\title{
Crocetin alleviates myocardial ischemia/reperfusion injury by regulating inflammation and the unfolded protein response
}

\author{
MING YANG ${ }^{1}$, GENXIANG MAO $^{2}$, LILI OUYANG ${ }^{3}$, CHENHUI SHI $^{1}$, PENGFEI HU $^{4}$ and SHUWEI HUANG ${ }^{4}$ \\ ${ }^{1}$ The Second Clinical Medical College, Zhejiang Chinese Medical University, Hangzhou, Zhejiang 310053; \\ ${ }^{2}$ Department of Geriatrics, Zhejiang Provincial Key Laboratory of Geriatrics and Geriatrics Institute of Zhejiang Province, \\ Zhejiang Hospital, Hangzhou, Zhejiang 310013; ${ }^{3}$ The Second Affiliated Hospital of Zhejiang Chinese Medical University; \\ ${ }^{4}$ Department of Cardiology, The Second Affiliated Hospital of Zhejiang Chinese Medical University, \\ Hangzhou, Zhejiang 310005, P.R. China
}

Received May 30, 2019; Accepted October 25, 2019

DOI: $10.3892 / \mathrm{mmr} .2019 .10891$

\begin{abstract}
Crocetin, a natural compound, has been demonstrated to exhibit beneficial effects in cardiovascular diseases. Previous studies demonstrated that crocetin reduced ischemia/reperfusion (I/R) injury by attenuating cytotoxicity and cellular apoptosis. However, the previous mechanistic studies did not fully elucidate its pharmacological effects on cardiac damage, especially $I / R$ injury. The present study verified its cardioprotective effects in a Langendorff perfusion system, an ex vivo model of $\mathrm{I} / \mathrm{R}$. It was demonstrated that crocetin significantly attenuated the activities of pro-inflammatory cytokines and nuclear factor erythroid-2 related factor 2 (Nrf2)/heme oxygenase-1 signaling. The present study provided novel insight that crocetin regulated the unfolded protein response (UPR) and decreased associated protein levels to protect the heart. Furthermore, it was identified that Nrf2 played a key role in the cardioprotective effect of crocetin by attenuating inflammation and the UPR.
\end{abstract}

\section{Introduction}

Cardiac vascular occlusion leads to the restriction of blood flow and oxygen to the heart, which causes cardiac ischemia. Although restoration of blood flow rescues the tissues from deficiency of oxygen and other supplementary metabolic products, subsequent lethal injury can induce myocardial damage and even death, termed ischemia/reperfusion $(I / R)$ injury $(1,2)$. Previous mechanistic studies demonstrated that calcium

Correspondence to: Professor Pengfei Hu or Professor Shuwei Huang, Department of Cardiology, The Second Affiliated Hospital of Zhejiang Chinese Medical University, 318 Chaowang Road, Hangzhou, Zhejiang 310005, P.R. China

E-mail: hupengfei5566@163.com

E-mail: hsw1104@126.com

Key words: crocetin, ischemia/reperfusion injury, inflammation, unfolded protein response, nuclear factor erythroid-2 related factor 2 overloading, mitochondrial-mediated apoptosis, redox disorder and inflammation play central roles in I/R injury (3-6). In the inflammatory response, the levels of pro-inflammatory cytokines, including interleukin (IL)-6, IL-18, IL-1 $\beta$ and tumor necrosis factor (TNF)- $\alpha$, markedly increased in the progress of cardiac I/R injury (7). The inflammatory response was induced quickly by cardiac I/R injury via the nuclear factor erythroid-2 related factor 2 (Nrf2) transcription factor-mediated signaling pathway (8). In Nrf2-deficient mice, the cardiovascular phenotype is more impaired, even in the ischemic preconditioning model, due to its important function in the cardiovascular system (9). Previous studies demonstrated that Nrf2/heme oxygenase-1 (HO-1) signaling plays an important role in I/R injury in different organs (10-13). Activation of $\mathrm{Nrf} 2 / \mathrm{HO}-1$ signaling is an effective method to attenuate myocardial I/R injury in several previous pharmacological studies $(14,15)$.

In cellular metabolism, the endoplasmic reticulum (ER) is responsible for protein folding to produce proteins with biologically functional structures for signal transduction. Extreme external stress disrupts protein folding and causes ER stress, which subsequently activates intracellular signaling transduction, termed the unfolded protein response (UPR) (16). Activating transcription factor 6 (ATF6), double-stranded RNA-activated protein kinase-like ER kinase (PERK) and inositol requiring enzyme 1 (IRE1), three UPR signal transducers, combine to function in glucose and lipid metabolism, leptin and insulin resistance, atherosclerosis and ischemia. Depending on these mechanisms, the UPR has been implicated in various physiological conditions, such as obesity, type 2 diabetes and I/R injury (17). Zhang et al (18) identified a critical role of the UPR; ER-induced cell apoptosis was observed under I/R injury and reversing the UPR could reduce the cardiac infarct size.

Crocetin (CRO), a natural apocarotenoid dicarboxylic acid is derived from Crocus sativus $L$., which originates from the Qinghai-Tibetan Plateau and can endure low concentrations of oxygen. Previous studies have demonstrated that CRO reduced cardiac cytotoxicity and apoptosis by regulating cardiac enzymes and their function (19,20). Additional previous studies demonstrated that $\mathrm{CRO}$ mechanistically regulated the mitogen associated protein kinase pathway and nuclear factor- $\kappa \mathrm{B}$ signaling 
to alleviate myocardial ischemia injury $(21,22)$. Moreover, CRO significantly reversed spatial learning dysfunction and attenuated hippocampal injury in an in vivo model of vascular dementia, which suggested that $\mathrm{CRO}$ exhibits more versatile functions than was previously known (23). A new study has been uncovered that another similar compound, Crocin, alleviates I/R injury by regulating ER stress and Nrf2/HO-1 signaling (24). Based on these previous studies, it was hypothesized that $\mathrm{CRO}$ could regulate Nrf2/HO-1 signaling and the UPR in the cardiovascular system. The aim of the present study was to evaluate whether CRO can protect the heart against I/R injury by alleviating inflammation via $\mathrm{Nrf} / \mathrm{HO}-1$ signaling and the UPR.

\section{Materials and methods}

Animals. Sprague Dawley rats (male, 3 months old, 250-300 g, total number: 120) were obtained from The Experimental Animal Center of Zhejiang Chinese Medical University (Lot: SCXK; Shanghai 2007-0005). The animals were acclimated for 7 days in a controlled temperature $\left(20-24^{\circ} \mathrm{C}\right)$ with 12 -h light/dark cycle and free access to food and water before the experiments. All experiments were designed according to The National Institutes of Health Guide for the Care and Use of Laboratory Animals and approved by The Animal Care Committee of Zhejiang Chinese Medical University. Every effort was made to minimize the number and suffering of the animals in the present study.

Langendorff perfusion and I/R injury. Rats were anesthetized with sodium pentobarbital $(50 \mathrm{mg} / \mathrm{kg})$ containing heparin (300 IU) by intraperitoneal injection. The rats were sacrificed and hearts were immediately harvested and mounted on the Langendorff system for retrograde perfusion at a constant pressure of $75 \mathrm{mmHg}$ with oxygenated $\left(95 \% \mathrm{O}_{2}\right.$ and $5 \%$ $\mathrm{CO}_{2}$ ) Krebs-Henseleit (KH) buffer $(118 \mathrm{mM} \mathrm{NaCl}, 4.7 \mathrm{mM}$ $\mathrm{KCl}, 1.2 \mathrm{mM} \mathrm{MgSO}_{4}, 1.25 \mathrm{mM} \mathrm{CaCl}, 1.2 \mathrm{mM} \mathrm{KH}_{2} \mathrm{PO}_{4}$, $25 \mathrm{mM} \mathrm{NaHCO}_{3}$ and $11 \mathrm{mM}$ glucose; $\mathrm{pH}$ 7.4) as previously described (25). Hemodynamic measurements for heart rate (HR), maximal rate of the increase of left ventricular pressure $\left(\mathrm{dp} / \mathrm{dt}_{\max }\right)$ and left ventricular developed pressure (LVDP) were assessed during the experiment. After $30 \mathrm{~min}$ for system equilibration, cardiac I/R injury was determined by the hearts experiencing $30 \mathrm{~min}$ ischemia (no flow) and $120 \mathrm{~min}$ reperfusion. The CRO treatment group was subjected to 20 min equilibration followed by $10 \mathrm{~min}$ CRO administration before I/R injury.

Drugs and chemicals. CRO (P0352; purity $\geq 95 \%$ ) was purchased from Shanghai PureOne Biotechnology and dissolved in DMSO (100 mM) for storage, and then diluted in KH buffer before use. All the chemical reagents used in the present study were of analytical grade. Rats were divided into 5 groups ( $n=8$; other rats were used to verify the success of the model and to test the surgical procedure): i) Sham, surgery without occlusion; ii) I/R, surgery with $30 \mathrm{~min}$ occlusion, followed by $120 \mathrm{~min}$ reperfusion; iii) I/R + CRO $(10 \mu \mathrm{M})$; iv) $\mathrm{I} / \mathrm{R}+\mathrm{CRO}(20 \mu \mathrm{M})$; and v) $\mathrm{I} / \mathrm{R}+\mathrm{CRO}(40 \mu \mathrm{M})$.

Cell culture and small interfering RNA (siRNA) interference. The H9c2 cardiomyocyte cell line was obtained from the American Type Culture Collection (cat. no. CRL1446) and cultured in DMEM containing 10\% FBS (Gibco; Thermo Fisher Scientific, Inc.) in a humidified incubator $\left(5 \% \mathrm{CO}_{2}\right)$ at $37^{\circ} \mathrm{C}$. Control siRNA (cat. no. sc-37007; scrambled sequence) and Nrf2 siRNA (cat. no. sc-37049) was purchased from Santa Cruz Biotechnology, Inc., and used according to the manufacturer's protocol. H9c2 cells were transiently transfected with Nrf2 siRNA $(10 \mu \mathrm{M})$ using Lipofectamine ${ }^{\circledR} 3000$ (Invitrogen; Thermo Fisher Scientific, Inc.) in opti-MEM (Gibco; Thermo Fisher Scientific, Inc.) for 6-12 $\mathrm{h}$. Then, the medium was replaced with DMEM for the following $48 \mathrm{~h}$.

Infarct size. The cardiac infarct size was determined by 2,3,5-triphenyltetrazolium chloride (TTC) staining, as previously described (26). The heart was frozen at $-20^{\circ} \mathrm{C}$ for $15 \mathrm{~min}$, then sliced into five $2 \mathrm{~mm}$-thick transverse sections and immersed in $1 \%$ TTC solution in distilled deionized water for $15 \mathrm{~min}$ at $37^{\circ} \mathrm{C}$ and then fixed in $4 \%$ paraformaldehyde overnight in $4^{\circ} \mathrm{C}$. The viable tissue was stained a deep red color and the infarcted zone was not stained. The infarcted size of each sliced heart section was measured and the percentage of the infarcted zone was calculated using ImageJ 1.48V (National Institutes of Health), image analyzing software.

Creatine kinase (CK), lactate dehydrogenase ( $L D H)$, superoxide dismutase (SOD), malondialdehyde (MDA) and GSH-PX (glutathione peroxidase). SOD, CK, LDH, MDA and GSH-PX production from the coronary flow were measured using a commercial kit (Nanjing Jiancheng Bioengineering Institute) according to the manufacturer's protocol. In brief, after I/R injury, the coronary blood flow was collected for SOD, CK, LDH, MDA and GSH-PX determination. The activities of the control group were considered as 1.

Reverse transcription ( $R T)-P C R$. The heart tissue was lysed and homogenized in $400 \mu 1$ lysis buffer (RLT Buffer; Qiagen, Inc.). The total RNA of the tissue was isolated on spin columns with silica-based membranes (RNeasy Mini kit; Qiagen, Inc.) according to the manufacturer's protocol. Then, RNA was reverse transcribed $\left(25^{\circ} \mathrm{C}\right.$ for $10 \mathrm{~min}$, following at $50^{\circ} \mathrm{C}$ for $15 \mathrm{~min}$, terminate the reaction by heating at $85^{\circ} \mathrm{C}$ for $5 \mathrm{~min}$ ) in a total volume of $20 \mu \mathrm{l}$ using the RT High-Capacity RNA-to-cDNA kit (Applied Biosystems; Thermo Fisher Scientific, Inc.). Quantitative PCR was performed with the FastStart Universal SYBR-Green Master Rox (Roche Diagnostics) using the ViiA ${ }^{\mathrm{TM}} 7$ real-time PCR system. The cycling protocol was as follows: $95^{\circ} \mathrm{C}$ for $2 \mathrm{~min}$, followed by 40 cycles at $95^{\circ} \mathrm{C}$ for $10 \mathrm{sec}$ and $60^{\circ} \mathrm{C}$ for $40 \mathrm{sec}$. All data were normalized to the housekeeping gene and the relative expression levels were calculated using the $2^{-\Delta \Delta \mathrm{Cq}}$ method (27). Primer sequences for IL-1 $\alpha$, IL-1 $\beta$, IL-6, IL- 8 and TNF- $\alpha$ were used to quantify the mRNA levels, as well as $\beta$-actin used as an endogenous control. The primer sequences are listed in Table I.

Cell viability. Cell viability was determined by evaluating the absorbance of MTT. Cells were cultured in 96-well microplates at a density of $1 \times 10^{4}$ cells $/ \mathrm{ml}$. After siRNA interference and $\mathrm{I} / \mathrm{R}$ injury, cells were incubated with medium containing MTT $(500 \mu \mathrm{g} / \mathrm{ml})$ for $3-4 \mathrm{~h}$ in the dark. Then, the MTT solution was aspirated and DMSO $(150 \mu \mathrm{l})$ was added for $15 \mathrm{~min}$. The absorbance was detected at $570 \mathrm{~nm}$ on a Multi-Mode Detection 
Table I. Primer sequences of IL- $1 \alpha$, IL-1 $\beta$, IL-6, IL-8, TNF- $\alpha$ and $\beta$-actin.

\begin{tabular}{lll}
\hline Name & \multicolumn{1}{c}{ Forward } & \multicolumn{1}{c}{ Reverse } \\
\hline IL-1 $\alpha$ & 5'-CCTCGTCCTAAGTCACTCGC-3' & 5'-GGCTGGTTCCACTAGGCTTT-3' \\
IL-1 $\beta$ & 5'-GCACAGTTCCCCAACTGGTA-3' & 5'-AAGACACGGGTTCCATGGTG-3' \\
IL-6 & 5'-CCACCCACAACAGACCAGTA-3' & 5'-GGAACTCCAGAAGACCAGAGC-3' \\
IL-8 & 5'-CTGCGCCAACACAGAAATTA-3' & 5'-ATTGCATCTGGCAACCCTAC-3' \\
TNF- $\alpha$ & 5'-CAGAGGGAAGAGTTCCCCAG-3' & 5'-CCTTGGTCTGGTAGGAGACG-3' \\
$\beta$-actin & 5'-GCTACAGCTTCACCACCACA-3' & 5'-ATCGTACTCCTGCTTGCTGA-3'
\end{tabular}

TNF, tumor necrosis factor; IL, interleukin.

Platform (SpectraMax Paradigm; Molecular Devices, LLC). Cell viability of the control group was considered as $100 \%$.

Immunoblot analysis. Heart tissue was homogenized in ice-cold RIPA buffer (Cell Signaling Technology, Inc.) containing protease inhibitors and incubated on ice for $20 \mathrm{~min}$, followed by centrifugation at $13,000 \mathrm{x}$ for $10 \mathrm{~min}$ at $4^{\circ} \mathrm{C}$. The supernatant was collected and the concentration of protein was quantified using the Bio-Rad protein assay kit (Bio-Rad Laboratories, Inc.). Proteins (30 $\mu \mathrm{g}$ /lane) of different groups were loaded and separated by SDS-PAGE on $10 \%$ gels and transferred to a PVDF membrane (pore size: $0.45 \mu \mathrm{m}$; EMD Millipore). The membranes were blocked with $5 \%$ bovine serum albumin in TBS with $0.1 \%$ Tween 20 (TBST) for $1 \mathrm{~h}$ at room temperature and incubated with $\operatorname{Nrf} 2(1: 1,000$, Cell Signaling Technology, Inc.; cat no. 12721), HO-1 (1:1,000, Cell Signaling Technology, Inc.; cat. no. 86806), PERK (1:500, Cell Signaling Technology, Inc.; cat. no. 5683), phosphorylated (p)-PERK (1:500, Cell Signaling Technology, Inc.; cat. no. 3179), IRE1 (1:1,000, Cell Signaling Technology, Inc.; cat. no. 3294), X-box binding protein 1 (XBP1; 1:1,000, Cell Signaling Technology, Inc.; cat no. 40435) and ATF6 (1:1,000; Cell Signaling Technology, Inc.; cat no. 65880) primary antibodies overnight at $4^{\circ} \mathrm{C}$. $\beta$-Actin $(1: 10,000$; Cell Signaling Technology, Inc.; cat no. 3700) was used as the loading control. After the primary antibody incubation, the membrane was washed with TBST 3 times ( $5 \mathrm{~min}$ ) and incubated with the anti-mouse and anti-rabbit IgG HRP secondary antibodies (1:10,000; Cell Signaling Technology, Inc.; cat. nos. 7076, 7074) for $1 \mathrm{~h}$ at room temperature. The intensity of the bands was detected using the ChemiDoc Touch Imaging System (Bio-Rad Laboratories, Inc.; series no. 732BR2237).

Statistical analysis. All data are presented as the mean \pm standard error of the mean with GraphPad Prism 7.0 (GraphPad Software, Inc.). The Student's t-test was used to analyze the differences between 2 groups and one-way analysis of variance with multiple comparisons (using Tukey's test) was used to analyze the differences between 3 or more groups. $\mathrm{P}<0.05$ was considered to indicate a statistically significant difference.

\section{Results}

$C R O$ alleviates myocardial $I / R$ injury. The present study established a Langendorff perfusion system, an ex vivo model of $\mathrm{I} / \mathrm{R}$, to evaluate the cardiac protective effect of CRO in rat hearts. $\mathrm{CRO}$ was dissolved in perfusion $\mathrm{KH}$ buffer and administered for $10 \mathrm{~min}$ before $30 \mathrm{~min}$ ischemia followed by 120 min reperfusion as shown in Fig. 1A. During the whole process of the experiment, cardiac functional parameters were recorded and analyzed. Long-term I/R injury decreased the HR, $\mathrm{dp} / \mathrm{dt}_{\max }$ and LVDP. CRO $(40 \mu \mathrm{M})$ markedly enhanced these cardiac functional parameters in the period of reperfusion, suggesting that $\mathrm{CRO}$ exhibited cardioprotection in rats (Fig. 1B-D).

After I/R injury, the heart was harvested for evaluation of the infarct size and the perfused buffer from the coronary flow was collected for determination of cardiac enzyme activities. A significant increase in the infarcted area was observed in the I/R group, $\sim 20 \%$ compared with the sham group $(\mathrm{P}<0.001)$. Dose-dependent decreases in infarct size were identified in the CRO pretreatment group, particularly in the CRO 40 group in which the infarct size was $<10 \%$ (Fig. $2 \mathrm{~A}$ and B). Then, the activities of two enzymes, $\mathrm{CK}$ and $\mathrm{LDH}$, were analyzed, which represented cardiac toxicity in the perfused buffer from the coronary flow. Both CK and LDH displayed downregulation in I/R hearts pretreated with CRO, suggesting that CRO could reduce cardiac toxicity from I/R injury (Fig. $2 \mathrm{C}$ and D). The antioxidant effect of CRO was further evaluated by determining the activities of SOD, MDA and GSH-PX. The changes of these three enzymes were significant in the I/R group and $\mathrm{CRO}$ regulated their activities in a dose-dependent manner $(\mathrm{P}<0.001$; Fig. 2E-G). Collectively, these observations demonstrated that $\mathrm{CRO}$ exhibited a protective effect to alleviate cardiac I/R injury.

CRO alleviates myocardial I/R injury via regulation of inflammation. As the inflammatory response accelerated tissue injury in the reperfusion period after the lethal ischemic damage, the levels of pro-inflammatory cytokines were detected. Cardiac mRNA levels of IL- $1 \alpha$, IL-1 $\beta$, IL-6, IL-18 and TNF- $\alpha$ significantly increased in the $\mathrm{I} / \mathrm{R}$ group $(\mathrm{P}<0.001)$. CRO significantly reduced the mRNA expression of these pro-inflammatory cytokines in a dose-dependent manner ( $\mathrm{P}<0.001$; Fig. 3A). Nrf2 is sensitive to oxidative stress and activates the transcription of HO-1 and NAD(P)H: Quinine oxidoreductase 1 (NQO1) in I/R injury (15). Nrf2-mediated signaling plays a key role in the innate immune/inflammatory pathway and regulates pro-inflammatory biomarkers, including IL-1 and TNF- $\alpha$ (28). Due to the importance of $\mathrm{Nrf} 2 / \mathrm{HO}-1$ signaling in the 
A

\begin{tabular}{|c|c|c|c|}
\hline \multirow{2}{*}{$\begin{array}{l}\text { A } \\
\text { Sham }\end{array}$} & \multicolumn{3}{|c|}{ Equilibrium } \\
\hline & \multicolumn{3}{|c|}{$180 \mathrm{~min}$} \\
\hline & Equilibrium & Ischemia & Reperfusion \\
\hline \\
\hline & $30 \mathrm{~min}$ & $30 \mathrm{~min}$ & $120 \min$ \\
\hline & Equilibrium CRO & Ischemia & Reperfusion \\
\hline \multirow[t]{2}{*}{ CRO } & & & \\
\hline & $20 \mathrm{~min} 10 \mathrm{~min}$ & $30 \mathrm{~min}$ & $120 \mathrm{~min}$ \\
\hline
\end{tabular}

C

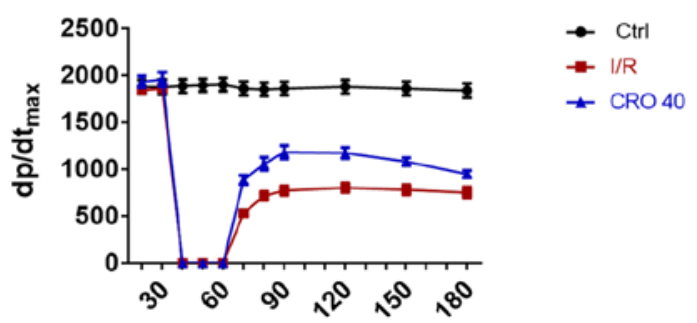

B

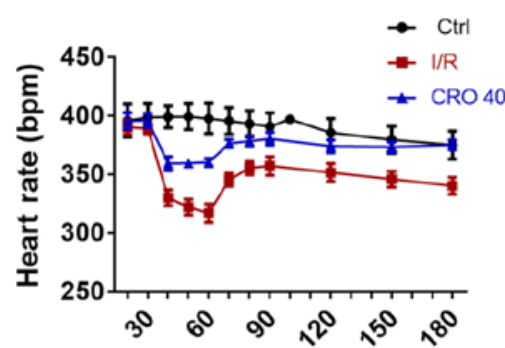

D

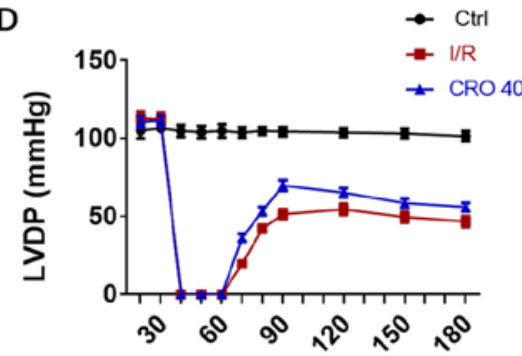

Figure 1. Cardiac protective effect of CRO in the Langendorff perfusion system. (A) Schematic representation of the experimental protocol. Sham: No occlusion; I/R: Surgery with $30 \mathrm{~min}$ occlusion, followed by $120 \mathrm{~min}$ reperfusion; CRO: $10 \mathrm{~min}$ pretreatment of CRO followed by I/R. Effect of CRO on cardiac functional parameters, including (B) heart rate, (C) $\mathrm{dp} / \mathrm{dt}_{\max }$ and (D) LVDP. Data $(\mathrm{n}=8)$ are presented as the mean \pm standard error of the mean. CRO, crocetin; $\mathrm{I} / \mathrm{R}$, ischemia/reperfusion; $\mathrm{dp} / \mathrm{dt}_{\max }$, maximal rate of the increase of left ventricular pressure; LVDP, left ventricular developed pressure; Ctrl, control.
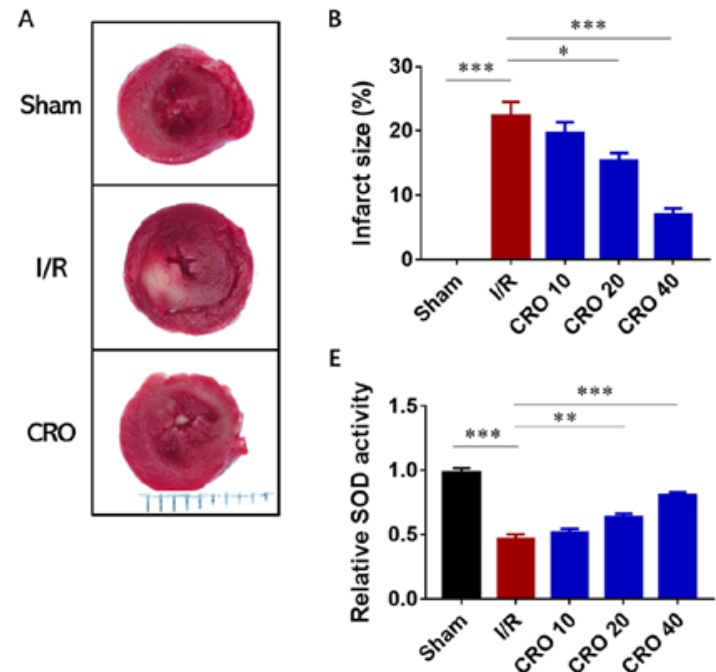
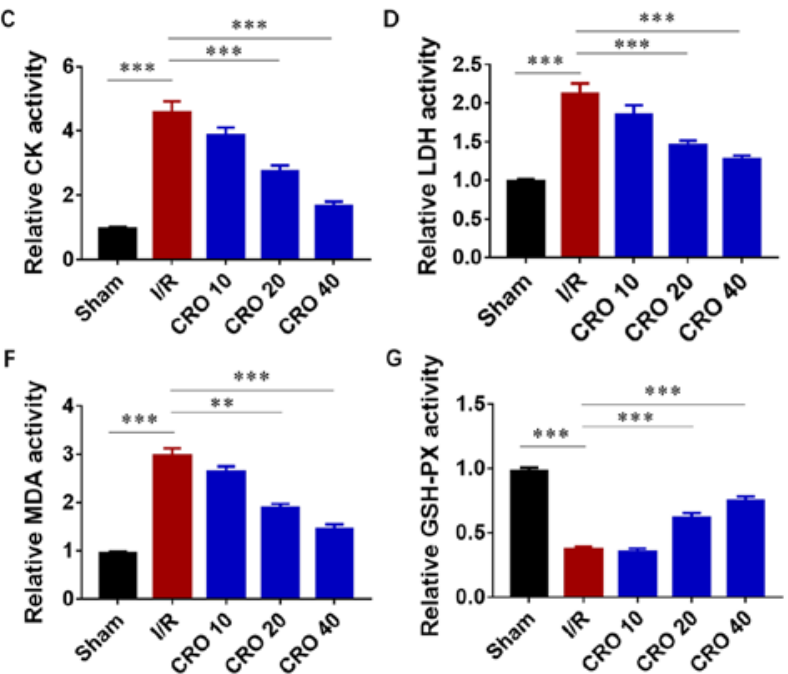

Figure 2. CRO alleviates myocardial I/R injury via regulation of cardiac enzyme activities. (A) Captured images and (B) analysis of the effect of CRO on the infarct size of the heart induced by I/R injury. Effect of CRO on (C) CK and (D) LDH of the heart induced by I/R injury. Effect of CRO on (E) SOD, (F) MDA and (G) GSH-PX of heart induced by $\mathrm{I} / \mathrm{R}$ injury. Data $(\mathrm{n}=8)$ are presented as the mean \pm standard error of the mean. ${ }^{*} \mathrm{P}<0.05$, ${ }^{* *} \mathrm{P}<0.01$ and ${ }^{* * *} \mathrm{P}<0.001$. CRO, crocetin; I/R, ischemia/reperfusion; CK, creatine kinase; LDH, lactate dehydrogenase; SOD, superoxide dismutase; MDA, malondialdehyde; GSH-PX, glutathione peroxidase.

inflammatory response induced by $\mathrm{I} / \mathrm{R}$, their protein expression was evaluated. I/R injury significantly reduced the protein expression of Nrf2, HO-1 and NQO1, and CRO upregulated their expression in a dose dependent fashion $(\mathrm{P}<0.001$; Fig. 3B). These results suggested that $\mathrm{CRO}$ could attenuate cardiac I/R injury via Nrf2/HO-1-mediated anti-inflammation signaling.

$C R O$ alleviates myocardial $I / R$ injury via regulation of $U P R$ signaling. The UPR is composed of three principle branches; IRE1, PERK and ATF6. The IRE1 branch is the central branch of the UPR; its activation cleaves the mRNA of XBP-1 and activates XBP-1 to translocate to the nucleus for transcription of genes involved in ER stress $(16,17)$. The UPR has been demonstrated to become activated when myocardial reperfusion occurs. Drugs with cardioprotective effects are demonstrated to attenuate the toxic UPR (29). Due to the important role of the UPR in I/R injury, immunoblot analysis was performed to evaluate the expression of proteins in the UPR system. The results showed that expression of p-PERK, IRE1, ATF6 and XBP-1 significantly increased in cells induced by I/R, suggesting I/R injury activated ER-stress signaling $(\mathrm{P}<0.001$; Fig. 4). Pretreatment with CRO significantly decreased the expression levels of these proteins, displaying a beneficial effect by downregulating ER-stress signaling (P<0.05; Fig. 4). 
A

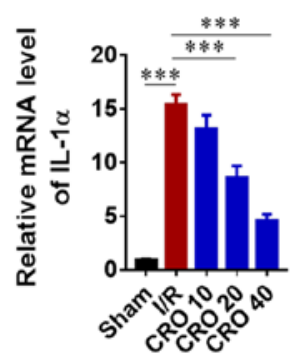

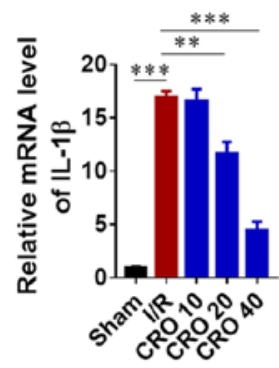
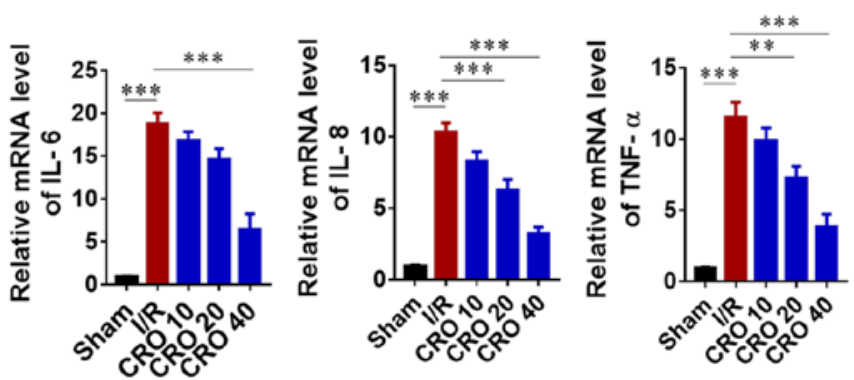

B
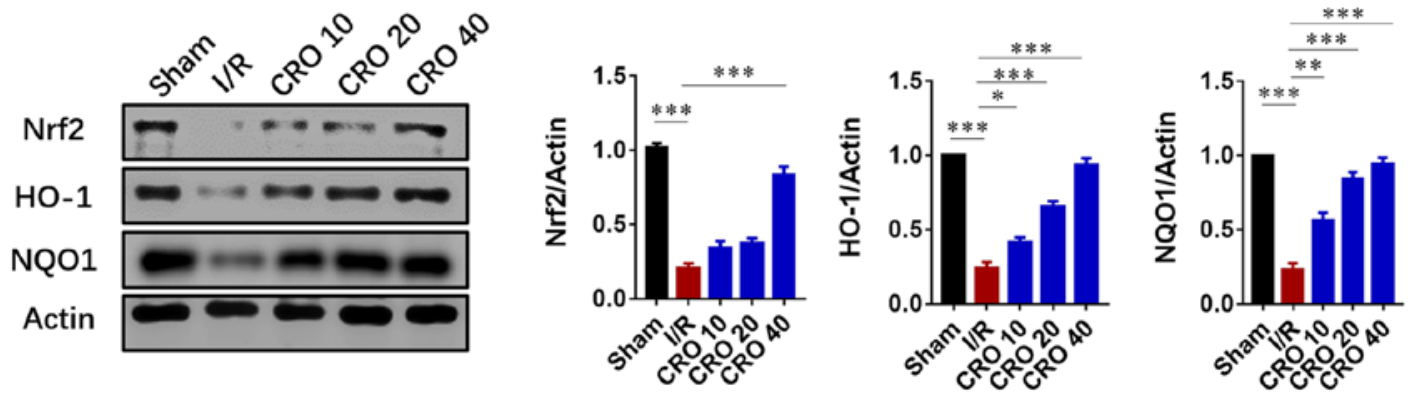

Figure 3. CRO alleviates myocardial I/R injury via regulation of the inflammation response. (A) Effect of CRO on pro-inflammatory cytokines of the heart induced by I/R injury. (B) Effect of CRO on Nrf2, HO-1 and NQO1 of the heart induced by I/R injury. Data $(\mathrm{n}=8)$ are presented as the mean \pm standard error of the mean. " $\mathrm{P}<0.05,{ }^{* * *} \mathrm{P}<0.01$ and ${ }^{* * *} \mathrm{P}<0.001$. CRO, crocetin; $\mathrm{I} / \mathrm{R}$, ischemia/reperfusion; UPR, unfolded protein response; Nrf2, nuclear factor erythroid-2 related factor 2; HO-1, heme oxygenase-1; NQO1, NAD(P)H: quinine oxidoreductase 1.

CRO alleviates myocardial I/R injury possibly in a Nrf2-dependent manner. Nrf2 transcription activated antioxidant and anti-inflammatory gene expression against cardiac I/R injury. Therefore, it was further evaluated whether CRO protected the heart against I/R injury in a Nrf2-dependent manner. An in vitro system was first applied using Nrf2 siRNA to downregulate Nrf2 expression in $\mathrm{H} 9 \mathrm{c} 2$ cardiomyocytes. The results showed that both the protein and mRNA expression reduced $>1 / 2$ after 54-60 h siRNA treatment (Fig. 5A and B). Subsequently, it was investigated whether downregulation of UPR protein expression by CRO pretreatment was dependent on Nrf2 activation. In the group with control siRNA (non-target) treatment, CRO pretreatment significantly reduced the expression of IRE1, ATF6 and XBP1. However, in the group with Nrf2 siRNA, the expression of IRE1, ATF6 and XBP1 did not reduce even with $\mathrm{CRO}$ pretreatment, suggesting that $\mathrm{CRO}$ attenuated the I/R-induced UPR in a Nrf2-dependent manner (Fig. 5C). The mRNA levels of pro-inflammatory cytokines were also measured and the results clearly showed that deficiency of Nrf2 suppressed the anti-inflammatory ability of CRO (Fig. 5D). From these results, it was determined whether reduction of $\mathrm{Nrf} 2$ expression inhibited the cardioprotective effect of CRO. An MTT assay was performed to evaluate cell survival and the results showed that CRO significantly increased cell viability in $\mathrm{H} 9 \mathrm{c} 2$ cells induced by I/R. In the group with Nrf2 siRNA, CRO could not enhance cell survival, suggesting that the cardioprotective effect of CRO required Nrf2 activation (Fig. 5E).

\section{Discussion}

The present study provided insight into the cardioprotective effect of CRO, a natural compound and its functional mechanisms. A classical ex vivo model of cardiac $\mathrm{I} / \mathrm{R}$ injury was used, termed the Langendorff perfusion system, to establish a working experimental evaluation system. The limitation of the animal experiment is lack of an in vivo pharmacological study. In further pharmacological evaluation, the authors are going to perform I/R injury in a whole animal and study the protective effect and concentration of CRO. Moreover, histological analysis of an in vivo sample can also be collected to evaluate the pathological parameters.

Classical mechanical hypotheses suggest that CRO protected against I/R injury in the brain and heart via an antioxidant response. However, previous studies demonstrated that inflammation served an important role in reperfusion injury, causing a secondary cascade of lethal damage $(2,12)$. Therefore, it was suggested that $\mathrm{CRO}$ had the ability to reduce the inflammatory response induced by reperfusion injury. In recent years, the UPR system, also mitochondrial UPR, has become a very important cellular adaptive system to regulate the stress-induced signaling pathway, especially in $\mathrm{I} / \mathrm{R}$ injury $(18,30)$. Based on the beneficial effect of CRO to the heart and previous references (21-23), it was hypothesized that CRO can regulate the inflammation response and the UPR system.

The present results demonstrated that $\mathrm{CRO}$ upregulated Nrf2/HO-1 signaling to reduce the inflammation response and downregulated UPR protein activities. As Nrf2 is a transcription factor, it activates a series of anti-inflammatory molecules and a previous study showed loss of $\mathrm{Nrf} 2$ reduced the activities of ER stress-related protein expression (24). Therefore, siRNA was used to downregulate $\mathrm{Nrf} 2$ expression, to determine whether Nrf 2 was essential for CRO to display its cardiac protective effects. The present results found that the cardioprotective effect of CRO was reduced in Nrf2-deficient cells, indicating that CRO protected cardiac functions in a Nrf2-dependent manner, including its regulation of UPR signaling. However, the relationship between the Nrf2-mediated inflammatory 
A

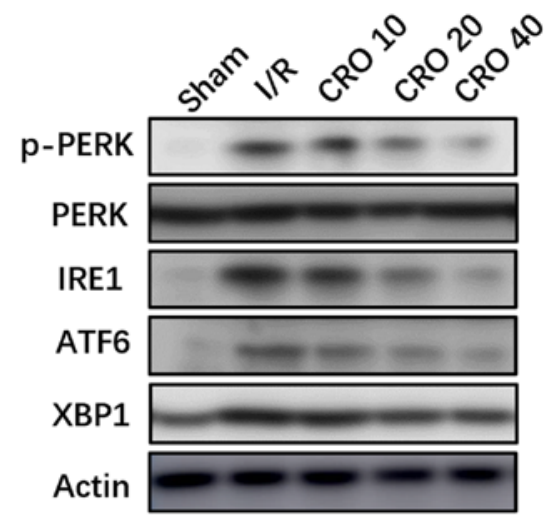

B
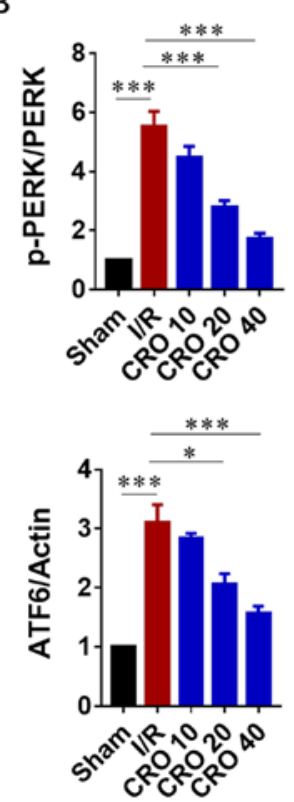
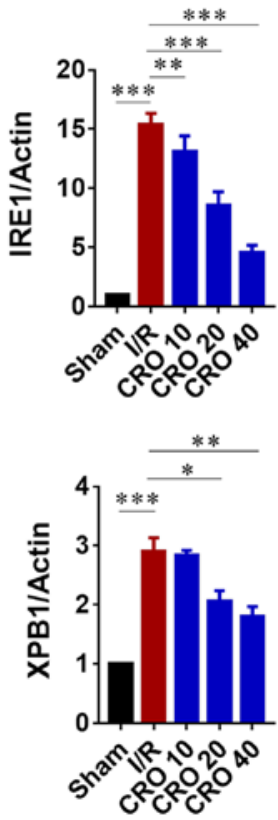

Figure 4. CRO alleviates myocardial I/R injury via regulation of UPR. (A) Western blotting and (B) analysis of the effect of CRO on UPR signaling proteins of the heart induced by $\mathrm{I} / \mathrm{R}$ injury. Data $(\mathrm{n}=8)$ are presented as the mean \pm standard error of the mean. ${ }^{*} \mathrm{P}<0.05,{ }^{* * *} \mathrm{P}<0.01$ and ${ }^{* * * *} \mathrm{P}<0.001$. CRO, crocetin; $\mathrm{I} / \mathrm{R}$, ischemia/reperfusion; UPR, unfolded protein response; p-PERK, phosphorylated-protein kinase-like ER kinase; IRE1, inositol requiring enzyme 1; ATF6, Activating transcription factor 6; XBP1, X-box binding protein 1.

A

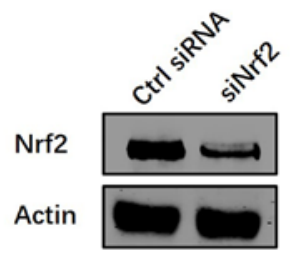

B

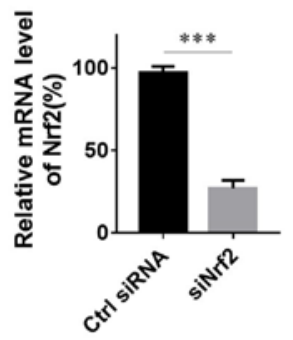

D

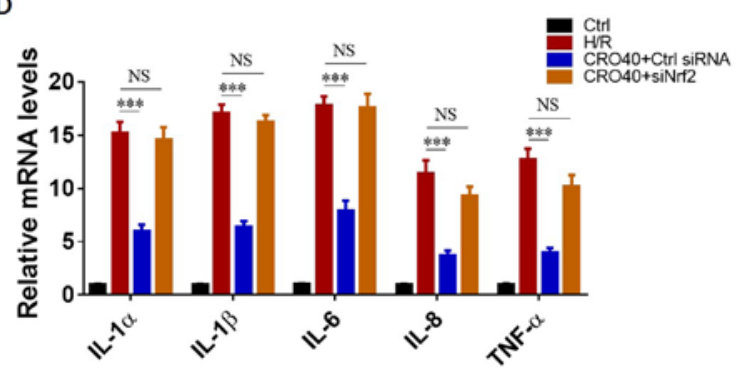

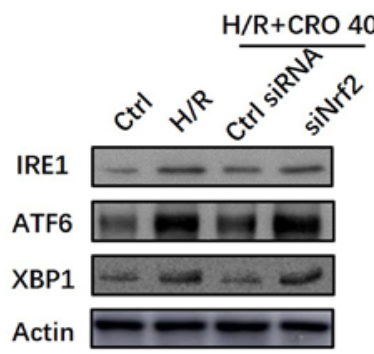

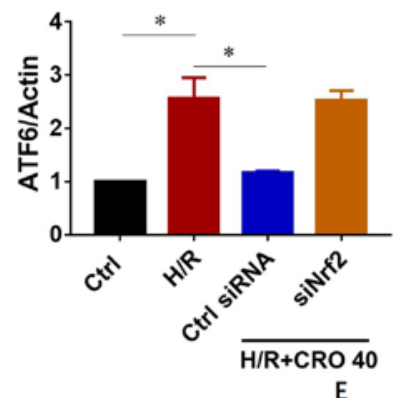

E
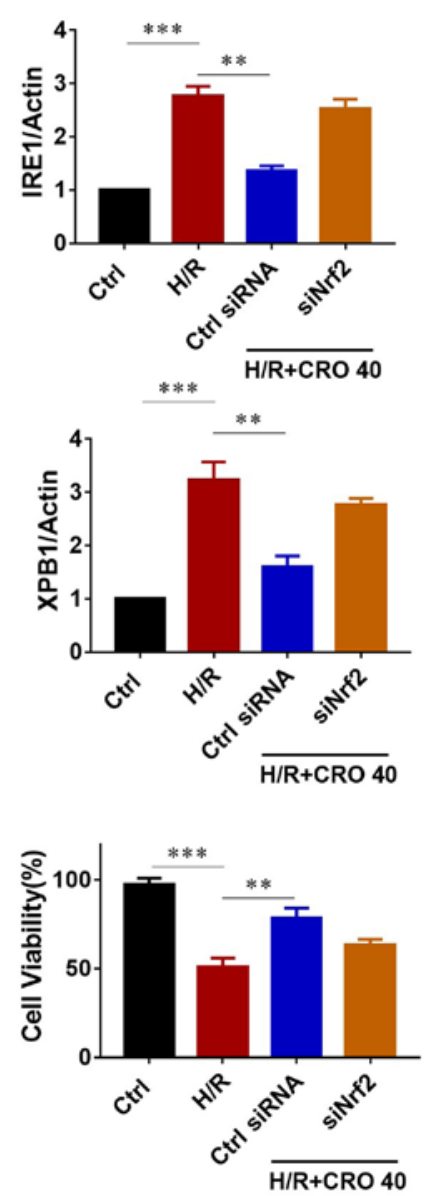

Figure 5. CRO alleviates myocardial I/R injury possibly in a Nrf2-dependent manner. (A and B) Effect of Nrf2 siRNA on protein and mRNA expression of Nrf2 in H9c2 cells. (C) Nrf2 siRNA treatment reduced the effect of CRO on UPR protein expressions. (D) Nrf2 siRNA treatment reduced the effect of CRO on pro-inflammatory cytokinases. (E) Nrf2 siRNA treatment reduced the effect of CRO on cell survival. Data $(\mathrm{n}=6)$ are presented as the mean \pm standard error of the mean. ${ }^{*} \mathrm{P}<0.05,{ }^{* *} \mathrm{P}<0.01$ and ${ }^{* * *} \mathrm{P}<0.001$. CRO, crocetin; I/R, ischemia/reperfusion; Nrf2, nuclear factor erythroid-2 related factor 2 ; siRNA, small interfering RNA; UPR, unfolded protein response; NS, no significance. 
response and UPR signaling remains unknown. Additionally, further investigation is required to elucidate the underlying mechanism of CRO and its Nrf2-specific binding domains and whether overexpressed Nrf2 may protect the heart from I/R injury via downregulation of UPR activation or conversely, whether downregulation of UPR activation may affect Nrf2/HO-1 signaling activation by CRO. Future studies may consider whether CRO could be applied to treat other cardiomyopathies and reperfusion-induced cellular toxicity.

\section{Acknowledgements}

Not applicable.

\section{Funding}

The present study was supported by grants from The Traditional Chinese Medicine Key Research Project (grant no. 2014ZZ004) and The National Natural Science Foundation of China (grant no. 81771520).

\section{Availability of data and materials}

All data generated or analyzed during this study are included in this published article.

\section{Authors' contributions}

MY designed and finished most of the experiments of this project. GM provided general support in experiments and analyzed part of data. LO and CS helped to feed and prepare animals, and also performed some of the biochemical kit measurements. $\mathrm{PH}$ and $\mathrm{SH}$ provided funding, controlled the progress of this study and made substantial contributions to the conception of this work. In addition, SH drafted the manuscript.

\section{Ethics approval and consent to participate}

All experiments were designed according to The National Institutes of Health Guide for the Care and Use of Laboratory Animals and approved by The Animal Care Committee of Zhejiang Chinese Medical University.

\section{Patient consent for publication}

Not applicable.

\section{Competing interests}

The authors declare that they have no competing interests.

\section{References}

1. Frank A, Bonney M, Bonney S, Weitzel L, Koeppen M and Eckle T: Myocardial ischemia reperfusion injury: From basic science to clinical bedside. Semin Cardiothorac Vasc Anesth 16 123-132, 2012.

2. Yellon DM and Hausenloy DJ: Myocardial reperfusion injury. N Engl J Med 357: 1121-1135, 2007.

3. Dhalla NS, Elmoselhi AB, Hata T and Makino N: Status of myocardial antioxidants in ischemia-reperfusion injury. Cardiovasc Res 47: 446-456, 2000.
4. Bolli R: Cardioprotective function of inducible nitric oxide synthase and role of nitric oxide in myocardial ischemia and preconditioning: An overview of a decade of research. J Mol Cell Cardiol 33: 1897-1918, 2001.

5. Garlid KD, Dos Santos P, Xie ZJ, Costa AD and Paucek P. Mitochondrial potassium transport: The role of the mitochondrial ATP-sensitive $\mathrm{K}(+)$ channel in cardiac function and cardioprotection. Biochim Biophys Acta 1606: 1-21, 2003.

6. Murphy E and Steenbergen C: Mechanisms underlying acute protection from cardiac ischemia-reperfusion injury. Physiol Rev 88: 581-609, 2008.

7. Kingery JR, Hamid T, Lewis RK, Ismahil MA, Bansal SS, Rokosh G, Townes TM, Ildstad ST, Jones SP and Prabhu SD: Leukocyte iNOS is required for inflammation and pathological remodeling in ischemic heart failure. Basic Res Cardiol 112: 19, 2017.

8. Chen QM and Maltagliati AJ: Nrf2 at the heart of oxidative stress and cardiac protection. Physiol Genomics 50: 77-97, 2018.

9. Jakobs P, Serbulea V, Leitinger N, Eckers A and Haendeler J: Nuclear factor (Erythroid-Derived 2)-like 2 and thioredoxin-1 in atherosclerosis and ischemia/reperfusion injury in the heart. Antioxid Redox Signal 26: 630-644, 2017.

10. Ji Q, Gao J, Zheng Y, Liu X, Zhou Q, Shi C, Yao M and Chen X: Inhibition of microRNA-153 protects neurons against ischemia/reperfusion injury in an oxygen-glucose deprivation and reoxygenation cellular model by regulating $\mathrm{Nrf} 2 / \mathrm{HO}-1$ signaling. J Biochem Mol Toxicol 31, 2017.

11. Zu G, Zhou T, Che N and Zhang X: Salvianolic acid A protects against oxidative stress and apoptosis induced by intestinal ischemia-reperfusion injury Through activation of Nrf2/HO-1 pathways. Cell Physiol Biochem 49: 2320-2332, 2018.

12. Tong $\mathrm{F}$ and Zhou X: The Nrf2/HO-1 pathway mediates the antagonist effect of L-arginine on renal ischemia/reperfusion injury in rats. Kidney Blood Press Res 42: 519-529, 2017.

13. Wang J, Hu X and Jiang H: ERS-PERK signaling pathway-mediated Nrf2/ARE-HO-1 axis: A novel therapeutic target for attenuating myocardial ischemia and reperfusion injury. Int J Cardiol 203: 779-780, 2016.

14. Cheng L, Jin Z, Zhao R, Ren K, Deng C and Yu S: Resveratrol attenuates inflammation and oxidative stress induced by myocardial ischemia-reperfusion injury: Role of Nrf2/ARE pathway. Int J Clin Exp Med 8: 10420-10428, 2015.

15. Zeng X, Li J and Li Z: Ginsenoside Rd mitigates myocardial ischemia-reperfusion injury via $\mathrm{Nrf} 2 / \mathrm{HO}-1$ signaling pathway. Int J Clin Exp Med 8: 14497-1504, 2015.

16. Walter $\mathrm{P}$ and Ron D: The unfolded protein response: From stress pathway to homeostatic regulation. Science 334: 1081-1086, 2011.

17. Lee $J$ and Ozcan $U$ : Unfolded protein response signaling and metabolic diseases. J Biol Chem 289: 1203-1211, 2014.

18. Zhang C, Tang Y, Li Y, Xie L, Zhuang W, Liu J and Gong J: Unfolded protein response plays a critical role in heart damage after myocardial ischemia/reperfusion in rats. PLoS One 12: e0179042, 2017.

19. Zhang W, Li Y and Ge Z: Cardiaprotective effect of crocetin by attenuating apoptosis in isoproterenol induced myocardial infarction rat model. Biomed Pharmacother 93: 376-382, 2017.

20. Kalalinia F, Ghasim H, Amel Farzad S, Pishavar E, Ramezani M and Hashemi M: Comparison of the effect of crocin and crocetin, two major compounds extracted from saffron, on osteogenic differentiation of mesenchymal stem cells. Life Sci 208: 262-267, 2018.

21. Huang Z, Nan C, Wang H, Su Q, Xue W, Chen Y, Shan X, Duan J, Chen $\mathrm{G}$ and Tao W: Crocetin ester improves myocardial ischemia via Rho/ROCK/NF-kappaB pathway. Int Immunopharmacol 38: 186-193, 2016.

22. Ishizuka F, Shimazawa M, Umigai N, Ogishima H, Nakamura S, Tsuruma $\mathrm{K}$ and Hara $\mathrm{H}$ : Crocetin, a carotenoid derivative, inhibits retinal ischemic damage in mice. Eur J Pharmacol 703: 1-10, 2013.

23. Tashakori-Sabzevar F, Hosseinzadeh H, Motamedshariaty VS, Movassaghi AR and Mohajeri SA: Crocetin attenuates spatial learning dysfunction and hippocampal injury in a model of vascular dementia. Curr Neurovasc Res 10: 325-334, 2013.

24. Wang X, Yuan B, Cheng B, Liu Y, Zhang B, Wang X, Lin X, Yang $\mathrm{B}$ and Gong G: Crocin alleviates myocardial ischemia/reperfusion-induced endoplasmic reticulum stress via regulation of miR-34a/Sirt1/Nrf2 pathway. Shock 51: 123-130, 2019. 
25. Facundo HT, Carreira RS, de Paula JG, Santos CC, Ferranti R, Laurindo FR and Kowaltowski AJ: Ischemic preconditioning requires increases in reactive oxygen release independent of mitochondrial K+ channel activity. Free Radic Biol Med 40: 469-479, 2006.

26. Zhang Z, Liu Y, Ren X, Zhou H, Wang K, Zhang H and Luo P: Caffeoylquinic acid derivatives extract of erigeron multiradiatus alleviated acute Myocardial ischemia reperfusion injury in rats through inhibiting NF-KappaB and JNK activations. Mediators Inflamm 2016: 7961940, 2016.

27. Livak KJ and Schmittgen TD: Analysis of relative gene expression data using real-time quantitative PCR and the 2(-Delta Delta C(T)) method. Methods 25: 402, 2001.

28. Huang Y, Li W, Su ZY and Kong AN: The complexity of the Nrf2 pathway: Beyond the antioxidant response. J Nutr Biochem 26 $1401-1413,2015$
29. Takatori O, Usui S, Okajima M, Kaneko S, Ootsuji H, Takashima SI, Kobayashi D, Murai H, Furusho $\mathrm{H}$ and Takamura M: Sodium 4-phenylbutyrate attenuates myocardial reperfusion injury by reducing the unfolded protein response. J Cardiovasc Pharmacol Ther 22: 283-292, 2017.

30. Bi X,Zhang G, Wang X, Nguyen C, May HI,Li X, Al-Hashimi AA, Austin RC, Gillette TG and Fu G, et al: Endoplasmic reticulum chaperone GRP78 protects heart from ischemia/reperfusion injury through Akt activation. Circ Res 122: 1545-1554, 2018.

(i) (3) $($ This work is licensed under a Creative Commons Attribution-NonCommercial-NoDerivatives 4.0 International (CC BY-NC-ND 4.0) License. 